\title{
The Application of the Digital Visual Effect Technology in the Urban Image Advertising Videos
}

\author{
Cai Zhe \\ Jiangxi University of Science and Technology, Ganzhou, Jiangxi, 341000 China \\ askamail@qq.com
}

Keywords: digital visual effect technology; urban areas; special advertising videos

\begin{abstract}
The digital visual effect technology provides the visual spectacle that cannot be performed by the traditional images. With the assistance of the digital visual effect technology, urban image advertising videos can reach an unprecedented new height. This paper applies and studies AE expression, studies the workflow and setting of the simplified software, and compiles the independent script code procedure.
\end{abstract}

\section{Introduction}

The film \& television feature film is a good method to promote the direct, active and accurate corporate image, urban, individual and product image. Special advertising videos set up the urban image with the strong visual impact and shock power of images to display the unique regional cultural feature. This is called as the urban "visual business card". The urban image advertising videos, as the brand-new spread form, have already become the important means to promote and publicize in each city and improve reputation. The digital visual effect technology provides the visual spectacle that can't be performed by the traditional images for us. Under the assistance of the digital visual effect technology, urban image advertising videos can reach the unprecedented new height. This paper applies and studies AE expression, studies the workflow and setting of the simplified software, and compiles the independent script code procedure.

\section{The role of the digital visual effect technology in the urban advertising videos}

\subsection{The role of the digital visual effect}

In the photographing stage of the urban image advertising videos, the digital visual effect can compensate for the inadequacy of photography to capacity. For example, when the frame photography is insufficiently stable, we can use the stable digital visual effect in the lens to repair the frames to capacity and reduce the difficulty of photography. In the production, digital visual effect can be stored and invoked, but it has no need to be reproduced. By taking the texture in software as an example, if the next scene needs the same texture, the previous production can be directly used and the texture in the material library can be saved. In the latest urban image advertising video in Inchon, South Korea, it uses the digital visual effect technology to display the future technology city under construction-Inchon and utilizes the visual effect technology to display the new image of the future city under the current efforts. The digital technology of full 3D animation utilizes the digital visual effect technology to create the clean urban environment by using 3D software and road overpass growing like magic, making us feel that Inchon is growing and making us full of expectation on the future Inchon, shown in Figure 1.

In the Olympic bid advertising video 2020 in Tokyo, it uses the digital visual effect technology to simulate the small heart-shaped LOGO. The small animated icons are gathered into thousands of icons from two people, thus the frame is filled with miracle, as shown in Figure 2.

In the Olympic bid advertising video of Madrid, it shows the urban value for the Olympic Games to display the urban landscape in the panorama. The special effect is used to produce lots of flowing light of ray and particles to embody light and heat for people brought by the Olympic Games, as 
shown in Figure 3.

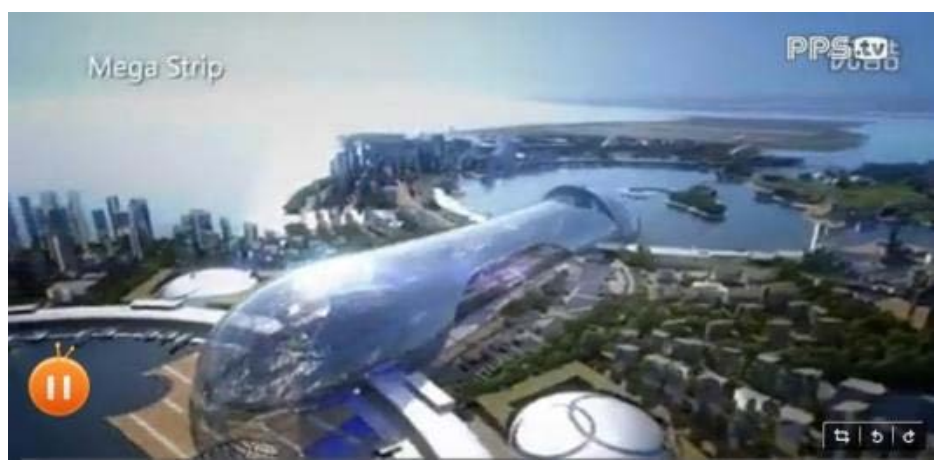

Fig.1 Inchon Advertising Video
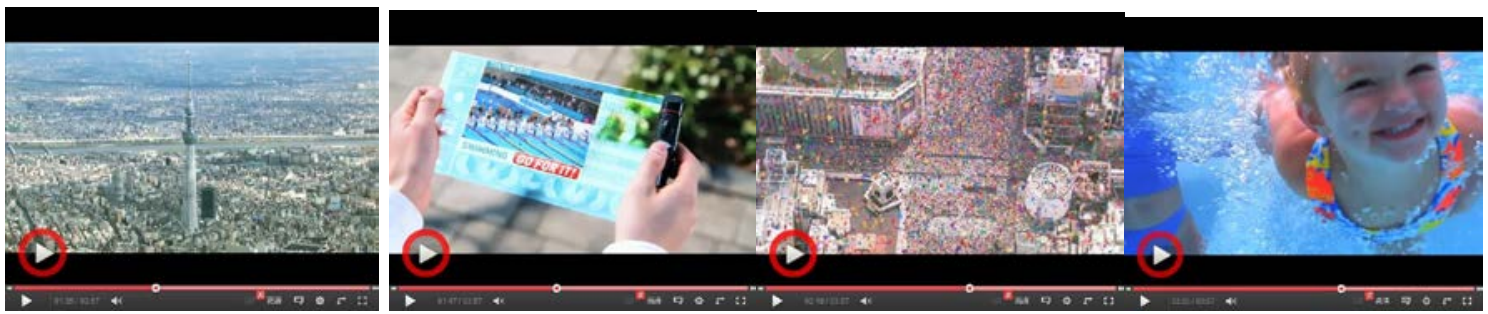

Fig.2 Advertising Video Data

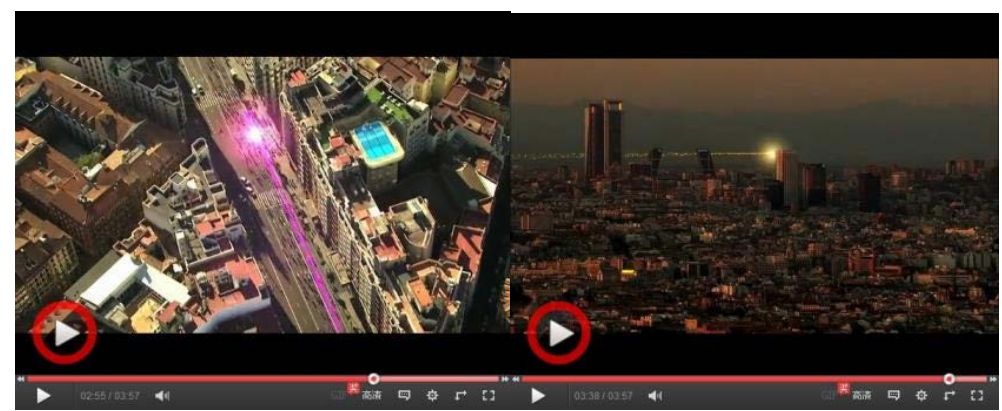

Fig.3 Advertising Video Data

\subsection{The symbol system of the urban image advertising videos}

The spread process of advertising videos uses the linguistic and non-linguistic symbols for urban image display. The essence is to display the beautiful urban images, thus the symbols have the representative and it is easy to comprehend. Hunan urban image advertising videos often apply the linguistic and non-linguistic symbols with the following types.

\begin{tabular}{|c|c|l|}
\hline \multirow{2}{*}{$\begin{array}{c}\text { Linguistic } \\
\text { symbols }\end{array}$} & Commentary & The entire commentary and non-entire commentary \\
\cline { 2 - 3 } & Characters & $\begin{array}{l}\text { Title and ending word, scenic spot notes, screen commentary } \\
\text { characters and other screen characters }\end{array}$ \\
\hline \multirow{2}{*}{$\begin{array}{c}\text { Non-linguistic } \\
\text { symbols }\end{array}$} & Body language & Figure action and facial expression \\
\cline { 2 - 3 } & Arts & $\begin{array}{l}\text { Music, dance, dieting features, urban buildings and cultural relics } \\
\text { and historic sites }\end{array}$ \\
\cline { 2 - 3 } & Others & $\begin{array}{l}\text { Urban facilities, natural environment, life state, folk custom and } \\
\text { custom habits }\end{array}$ \\
\hline
\end{tabular}

3. The application analysis of the digital special effect technology in urban image advertising videos

\subsection{The early-stage photography of the project}

The photography of early-stage materials is affected by external factors, such as season, weather 
and sunshine, and so on. It is necessary to select the sunny days to photograph. In this way, frame colors will be saturated and have the accurate exposure, thus the entire city is filled with the beautiful colors, as shown in Figure 4.
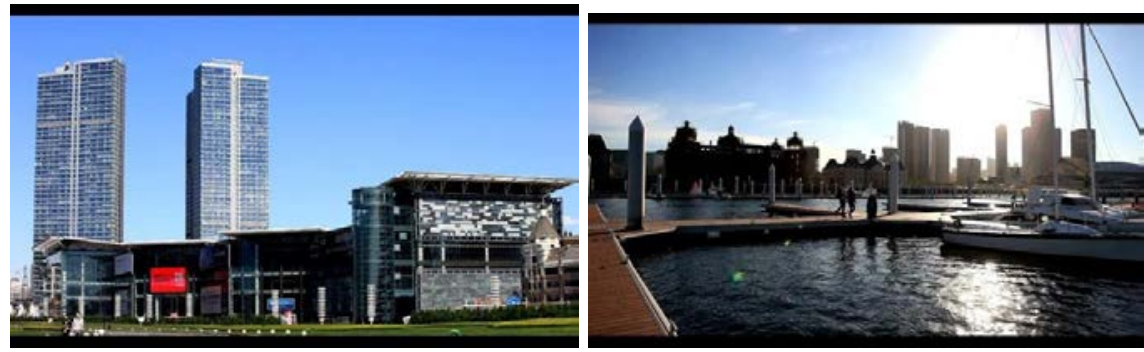

Fig.4 Urban Advertising Video Materials

Due to the production and synthesis of digital special effect technology in later period, the lens synthesized by special effect should be designed carefully and rigorously. We use the orbit in the photographing process to ensure the stable frame. In this way, it can save the trouble of frame stability in later-period production, save the working time and improve the working efficiency, as shown in Figure 5.
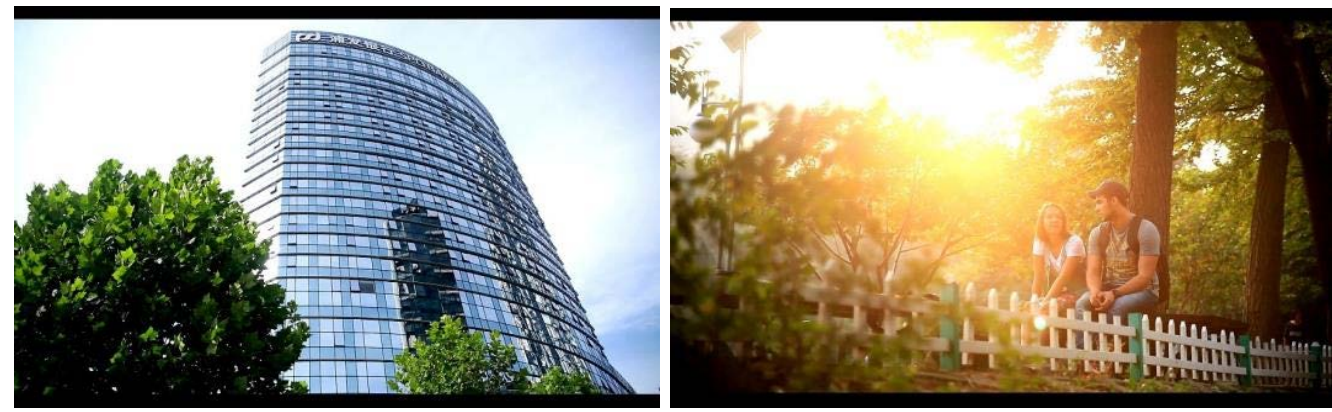

Fig.5 Urban Advertising Video Materials

\subsection{The special effect synthesis of the project}

The large process considers the $\mathrm{AE} 3 \mathrm{D}$ layer and sense of space. It not only displays the information conveyed by the videos, but also uses the $\mathrm{AE}$ position expression:

value + [effect ("Separate XYZPosition") ("X Position"), effect ("Separate XYZ Position”) ("Y Position"),

effect ("Separate XYZ Position”) ("Z Position")], to control the displacement animation of the lens, as shown in Figure 6.

Number or Array linear (t, value1, value2) $\{t$ is a number, value 1 and value 2 are a number or array\}, when t's range is $0-1$, the linear interpolation from value 1 to value 2 is returned. As $t<=0$, value 1 is returned. As $t>=1$, value 2 is returned. Number or array linear ( $t$, thin, tMax, value 1 , value2) $\{\mathrm{t}$, thin and tMax are the number, and value 1 and value 2 are the number or array $\}$. As $\mathrm{t}<=$ tmin, value 1 is returned; as $\mathrm{t}>=\mathrm{tMax}$, value 2 is returned. As thin $<\mathrm{t}<\mathrm{tMax}$, linear combination of value 1 and value 2 is returned. Number or array ease (t, value 1, value 2) \{t is a number. The value 1 and value 2 are the number of array\}. The return value is similar to linear. However, the rate of starting and ending point is 0 . Such a method result is animated and smooth.

Number or array ease ( $t$, thin, tMax, value1, value 2) $\{\mathrm{t}$, thin and tMax are the number, value 1 and value 2 are the number of the array\}. The return value is similar to finear, but the rate of starting and ending points is 0 . The method result is animated and smooth.

Number or array easeln(t, value 1 , value 2) $\{\mathrm{t}$ is a number, and value 1 and value 2 are the number of the array\}. The return value is similar to ease, but the rate at the entry point value 1 is. The side closing to value 2 is linear.

Number or array easeIn(t, thin, tMax, value 1, value 2) \{t, thin and tMax are a number, value 1 and value 2 are the number or array . The return value is similar to ease, but the rate at the entry point thin is 0 . The side closing to tMax is linear. 
Number or array ease0out ( $\mathrm{t}$, value 1, value 2) $\{\mathrm{t}$ is a number, value 1 and value 2 are the number or array\}. The return value is similar to ease, but only the rate at the entry point value 2 is. The side closing to value 1 is linear.

Number or array ease0out ( $t$, thin, tMax, value 1 , value 2$)$ \{t, thin and tMax are the number, value 1 and value 2 are the number or array\}. The return value is similar to ease, but only the rate at the entry point tMax is 0 . The side closing to thin is linear.

The following method can be used to write the position expression:

[position [0], position [1]];

After two parameters in the position array are respectively proposed, they are put into the same array. It seems that it has the same result with the written position, thus the $X$ value at the axis will be controlled and adjusted by AE, while $\mathrm{Y}$ value at the axis is always 0 .

In the production process of advertising videos, the early-stage scheme and conception are the most important and difficult. The perfect design and considerable overall reflection can dramatically improve efficiency. Otherwise, small negligence will bring a huge trouble to the project production. For example, as disposing materials, it is necessary to consider whether the project's pixel size is rational. Whether the large pixel size not only won't bring advantages, but also will make your project software collapse. For instance, as project nest, it needs to consider whether the project in different levels will add the special effect. the different adding levels of the same special effect will bring the different results.

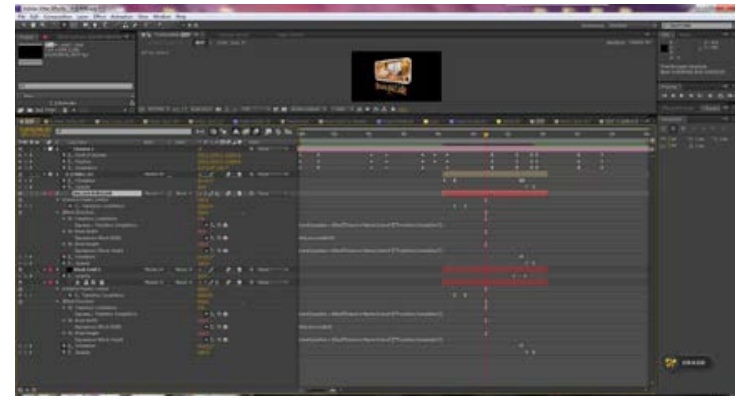

Fig.6 Special Effect Synthesis of the Urban Advertising Videos

\subsection{The expression applications of the urban image advertising videos}

Lots of digital special effect technologies in the urban image advertising videos can be performed and improved by using $\mathrm{AE}$ expression in the later-period synthesis software AE to speed up the production process. As executing the sentence, AE expression will automatically calculate the rotary parameter value of the current layer and the current time. The return value will endow the parameter value for expression connection. If the expression is applied in the non-transparent attribute of the layer, thus the non-transparent attribute of this layer will conduct synchronous change with the rotary attribute. As rotating at $30^{\circ}$, the non-transparent layer will be set up as $30 \%$.

A linear (t, thin, tMax, value 1 and value 2) function is used to translate the material out point on the time axis as the transparency measurement. The transparency expression is shown as follows:

If we conduct fade-in fade-out, "if...else..." judgment sentence is used to connect the above-mentioned two expressions, adding the transparent expression of materials as follows:

if $($ time $<1)$

linear (time, inPoint, inPoint+l,0, 100);

else

finear(time, outPoint-1,outPoint, 100, 0)

In this way, it realizes the material's fade-in and fade-out effect. Through RayCrown, the above-mentioned sentence has a loophole. When the fade-in point is moved to $1 \mathrm{sec}$, time $>1$, thus if won't be executed, but else will be executed.

RayCrown's standardized and standard sentence is shown as follows:

$\mathrm{s}=$ linear(time,inPoint,outPoint, value,value);

$\mathrm{a}=$ linear(time,inPoint,inPoint+l,-100, 0); 
b=linear (time,outPoint-1,outPoint, 0,-100);

$\mathrm{a}+\mathrm{b}+\mathrm{s}$

No matter how to adjust, the in-point and out-point animation is always consistent.

\section{Conclusions}

Lots of excellent urban image advertising videos have the very important significance on the urban propaganda and development, while digital special effect technology, as the brand-new potential artistic creation mode, definitely will greatly support the urban image advertising videos, thus creators can totally open the unrestrained imagination space and enjoy the creative space.

\section{References}

[1] Yu Zhong, the Study and Application of Digital Special Effect Technology in the Film Teaching[J], China Training, 2016(14): 173.

[2] Wan Jin, the Urban Advertising Video Photography and Later-period Special Effect Synthesis Study[J] Western Broadcasting TV, 2017(04): 102.

[3] Zhou Wanpeng, the Artistic Photography in Urban Advertising Videos[J], New Media Study, 2015, 1(14): 63+54.

[4] Liang Min and Yan He, “Xianyan Image” Spread Strategy Study under the Digitalization[J], News Study Magazine, 2015, 6(20): 35+29.

[5] Li Tiefeng, the Digital Stereoscopic Technology: New Fashion of the Film Media-the Discussion on Jinchang Digital Stereoscopic Urban Advertising Video[J], Broadcasting TV Information, 2011(01): 40-43. 